\title{
Optical Diagnostics of Temperature and Structural Parameters of an Axisymmetric Flame*
}

\author{
Vitaly Arbuzov ${ }^{1,3[0000-0003-2404-526 X]}$, Edward Arbuzov ${ }^{1,2,3[0000-0001-9488-8650], ~ Y u r i ~ D u b-~}$ \\ nishchev ${ }^{1,3[0000-0001-7874-039 X]}$, Olga Zolotukhina ${ }^{1,3[0000-0003-3486-4459]}$ and Vladimir \\ Lukashov ${ }^{10000-0001-8178-7607]}$ \\ ${ }^{1}$ Kutateladze Institute of Thermophysics, Siberian Branch of the Russian Academy of Sciences, \\ Novosibirsk 630090, Russia \\ ${ }^{2}$ Sobolev Institute of Mathematics, Siberian Branch of the Russian Academy of Sciences, No- \\ vosibirsk 630090, Russia \\ ${ }^{3}$ Novosibirsk State Technical University, Novosibirsk 630073, Russia \\ arbuzoveitp.nsc.ru|arbuzov@math.nsc.ruldubnistcheveitp.nsc.ru|me \\ lexina-olga17@ya.rulluka@itp.nsc.ru
}

\begin{abstract}
In this paper a method for restoring the parameters of multicomponent media for optical diagnostics of jet using the example of a hydrogen-air flame study is considered. Hilbert visualization and numerical modeling of phase perturbations induced by the studied medium in the probing light field are used. The study of the burning jet was carried out using the methods of Hilbert optics and Abel transformation in the model of axial symmetry of the torch. A software package has been developed that implements a direct solution to the problem: calculation of the spatial optical phase structure of the flame and its corresponding hilbertograms based on the temperature and molar concentrations of the combustion products of the mixture. The reliability of the obtained results is confirmed by comparing the Hilbert structures obtained in the experiment and the reconstructed optical density field of the phase using the Abel transform. The results of the comparison are used as a quality criterion for modeling the phase structure and temperature field in the study of the combustion process. The developed method can be used to solve the inverse problem of restoring the temperature field from the Hilbert image of the phase structure of a hydrogen-air flame in the approximation of axial symmetry. The research is motivated by the scientific and practical significance of the problem, which consists in finding methods for controlling the structural and thermodynamic parameters of reacting jets and flames.
\end{abstract}

Keywords: Optical Diagnostics of Flames, Hilbert Optics, Optical Information Technology.

Copyright (C) 2020 for this paper by its authors. Use permitted under Creative Commons License Attribution 4.0 International (CC BY 4.0).

* The work was done within the framework of the state assignment of IT SB RAS project III.18.2.5 (state registration No. AAAA-A17-117030310010-9, No. AAAA-A17117022850021-3), partial financial support from RFBR (project No. 20-38-90195). 


\section{Introduction}

The optical methods are the most important in unperturbing diagnostics of reacting jets and flames. They allow one to obtain adequate information about the thermodynamic and structural parameters of the medium under study without disturbing its state.

In [1], a method for estimating the temperature distribution in an asymmetric flame using high-contrast stereoscopic photography is described. Spectral reconstruction of temperature fields using color ratio pyrometry and interferometric tomography is reported [2]. An example adapted to the problems of flame research is optical diagnostics based on the methods of Hilbert optics and interferometry in combination with pixel-by-pixel processing of the dynamic structure of visualized phase structures induced by temperature fields [3]. However, the problem is to diagnose the spatial optical phase structure of the flame.

The aim of this work is to adapt the methods of Hilbert optics to the study of axisymmetric hydrogen-air flame with the reconstruction of the spatial phase and temperature structure. The research is motivated by the scientific and practical significance of the problem, which consists in finding methods for controlling the structural and thermodynamic parameters of the torch [4].

\section{Research method}

The complex of optical diagnostics is based on the IAB-463M device [5] with modified modules of Hilbert filtration, interferometry, source of probing field, registration and processing of the optical signal [6]. The probing field is formed by the collimator system from a slotted source. The investigated hydrogen-air flame is localized in the vicinity of the front Fourier plane of the lens, which serves as an analyzer of phase disturbances induced in the probing field by this flame. Visualization of the Fourier spectrum of phase perturbations is performed by a Hilbert filter placed in the frequency plane of the Fourier lens. Next, the inverse Fourier transform of the Hilbertconjugate Fourier spectrum of phase perturbations is performed. The visualized phase disturbances are registered by the camera's photo matrix. The registered images are analyzed by a computer connected to the video camera.

In the frequency plane for the Fourier spectrum of the phase optical density of the light field perturbed by the medium under study (flame), we have immediately after the filter:

$$
H\left(K_{x}, K_{y}\right) s\left(K_{x}, K_{y}\right)=s\left(K_{x}, K_{y}\right) \cos \varphi+\hat{s}_{x}\left(K_{x}, K_{y}\right) \sin \varphi,
$$

where $H\left(K_{x}, K_{y}\right)$ is the coherent transfer function of the filter performing the onedimensional Foucault-Hilbert transform, 
Optical Diagnostics of Temperature and Structural Parameters of an Axisymmetric Flame 3

$$
H\left(K_{x}, K_{y}\right)=\cos \varphi-i \sin \varphi \operatorname{sgn} K_{x} .
$$

The phase shift $\varphi$ is a function of the wavelength $\lambda$ of the probing light field, $\varphi=\varphi(\lambda)$. At a wavelength satisfying the condition $\varphi\left(\lambda_{0}\right)=\pi / 2$, the Hilbert image of the Fourier spectrum of phase perturbations:

$$
\hat{s}_{x}\left(K_{x}, K_{y}\right)=-i \operatorname{sgn} K_{x} s\left(K_{x}, K_{x}\right) .
$$

The inverse Fourier transform of the Hilbert-conjugate Fourier spectrum of phase perturbations (1) is performed below:

$$
s\left(K_{x}, K_{x}\right) \cos \varphi+\hat{s}_{x}\left(K_{x}, K_{x}\right) \sin \varphi \leftrightarrow s(x, y) \cos \varphi+\hat{s}_{x}(x, y) \sin \varphi .
$$

The intensity of the analytical signal (3) is recorded by a video camera:

$$
I(x, y)=\rho\left\{|s(x, y)|^{2} \cos ^{2} \varphi+\left|\hat{s}_{x}(x, y)\right|^{2} \sin ^{2} \varphi\right\},
$$

where $\rho$ is a coefficient that takes into account the sensitivity of the photo matrix.

The Hilbert transform has the properties of redistributing energy from the region of low spatial frequencies to the high-frequency region. Extremes and gradients of the phase optical density of the medium under study are transformed into visualized Hilbert-bands structures. The spatial distribution of Hilbert bands provides information about phase optical density perturbations induced by the temperature field.

An example of the phase structure of a hydrogen-air flame is shown in Fig. 1.a. The phase structure was visualized using Hilbert optics methods. The experiments were carried out at atmospheric pressure and initial room temperature.
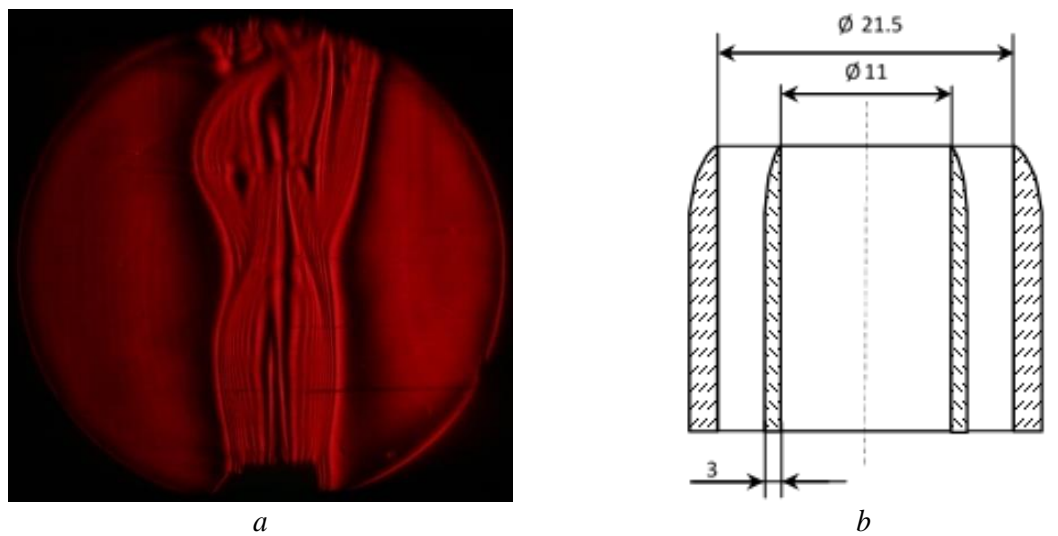

Fig. 1. (a) - experimental hilbertogram of a hydrogen-air flame; $(b)$ - is a diagram of the burner arrangement.

The burner is shown schematically in Fig. 1.b. It consists of two coaxially and vertically positioned quartz tubes (dimensions and relative positions are shown in the figure). Air is supplied through an inner tube. The fuel mixture $\mathrm{H}_{2} / \mathrm{N}_{2}$ enters through an 
annular channel between the inner and outer pipes $(36.51 \%$ by volume, $3.95 \%$ by mass). The nitrogen is added to reduce heat transfer to the burner. The diffuse flame research was performed in sections with axial symmetry. The choice of the area with axial symmetry is explained by the relatively simple geometry when modeling the flame.

Measurements of the radial temperature distribution in three sections of the flame at distances of 0,30, and $60 \mathrm{~mm}$ from the cut of tubes (Fig. 2) were completed in a mode corresponding to an average flow rate of air on the axis of $1 \mathrm{~m} / \mathrm{s}$ and an average flow rate of fuel of $0.8 \mathrm{~m} / \mathrm{s}$, using a platinum thermocouple.

According to the dispersion formula Cauchy $[7,8]$

$$
n_{k}(\lambda)-1=A_{k}\left(1+\frac{B_{k}}{\lambda^{2}}+\frac{C_{k}}{\lambda^{4}}\right),
$$

the refractive index of the $k$ th component of the burning mixture $n_{k}$ depends on the wavelength of the radiation source $\lambda$ and the parameters $A_{k}, B_{k}$ and $C_{k}\left(A_{k}\right.$ and $B_{k}$ are dispersion coefficients for the $k$ th component, $C_{k}$ is the relative molar concentration of the $k$ th component).The refractive index of the entire mixture is defined as

$$
n-1=\frac{p}{p_{0}} \frac{T_{0}}{T} \sum_{k} A_{k}\left(1+\frac{B_{k}}{\lambda^{2}}\right) \cdot C_{k},
$$

where $p$ is the pressure; $p_{0}$ - pressure (in the room); $T$ is the temperature; $T_{0}$ - room temperature. The reconstruction of the values of molar concentrations of fuel combustion products $C_{k}$ and, accordingly, the temperature distribution in the flame is possible by performing measurements at different wavelengths and using formulas (5) - (6).

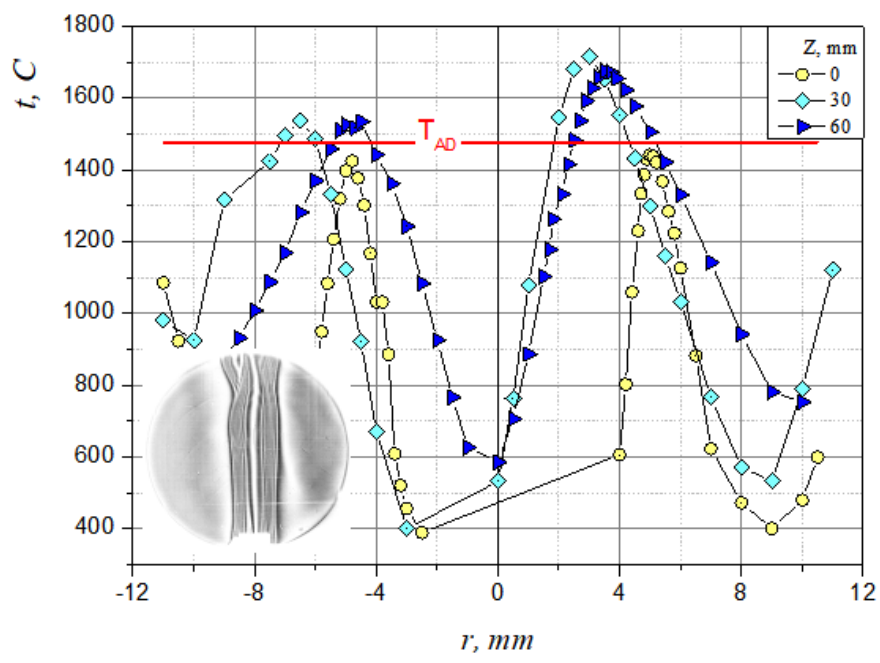

Fig. 2. The temperature distribution in three sections along the radius at heights from the cut of tubes of 0,30 and $60 \mathrm{~mm}$ at an average flow rate of air on the axis of $1 \mathrm{~m} / \mathrm{s}$ and an average flow rate of fuel of $0.8 \mathrm{~m} / \mathrm{s}$. 
An algorithm for the numerical simulation of Hilbert images by radial values of temperature and mole fraction of fuel combustion products in a hydrogen-air flame was developed. This algorithm was tested on the experimental data given in [9] (temperature profiles and molar concentrations in four sections of an axisymmetric flame section).

The graphs displaying the radial profiles of temperature, molar concentrations of fuel combustion products, and refractive index in the section at a height from the cut of the burner tube $y=10 \mathrm{~mm}$ are shown in Fig. 3.a - 3.b. The radial temperature field of an axisymmetric section of a hydrogen-air flame, obtained using piecewise-cubic Hermitian interpolation by the temperature values in sections at heights $y=3,10,20$, and $30 \mathrm{~mm}$, is shown in Fig. 4.

The phase structure of the probing light field, disturbed by the medium under study (flame), in a physical experiment is defined as:

$$
\Delta \psi(x, y)=k \int_{z_{1}}^{z_{2}}\left[n(x, y, z)-n_{0}\right] d z
$$

where $k=2 \pi / \lambda$ is the wave number of the probing field; $n(x, y, z)$ is the refractive index of the medium in the spatial structure of the flame; $n_{0}$ is the refractive index of the medium unperturbing by the flame. The $z$ axis is determined by the direction of the probe light beam, the flame torch cross section is described in $x, z$ coordinates. The choice of the section position is determined by the $y$ coordinate. The coordinates $z_{1}, z_{2}$ specify the size of the flame section in the direction of the probe beam.
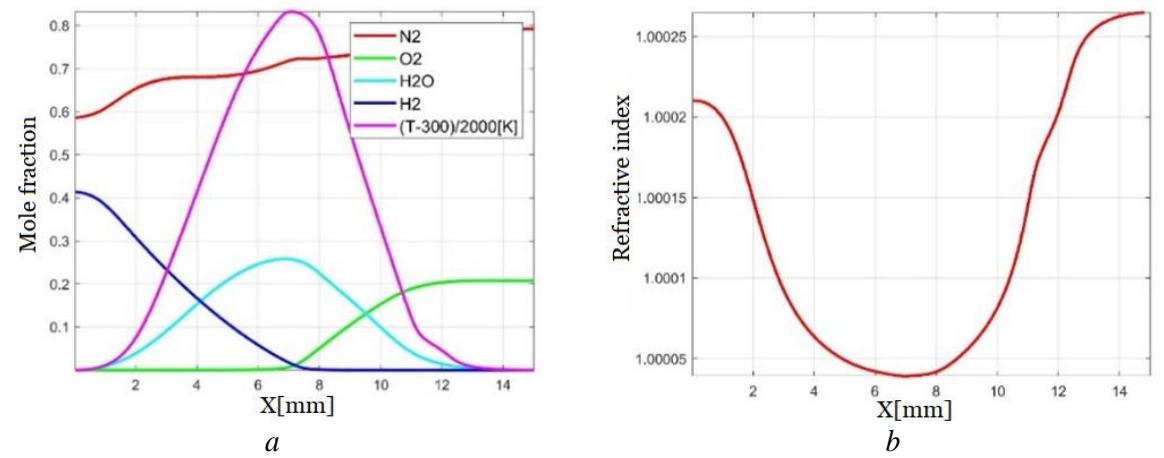

Fig. 3. (a) - radial profiles of temperature $T$ and molar concentrations $C_{k} ;(b)$ - radial profile of the refractive index $n(r)$, calculated for $\lambda=0.630 \mu \mathrm{m}(y=10 \mathrm{~mm}$ from the end of the burner tube cut).

Eq. (7) is turned into the Abel equation in the case of axial flame symmetry (Fig. 5):

$$
\Delta \psi(r, y)=2 k \int_{x}^{R}\left[n(r, y)-n_{0}\right] \frac{r d r}{\sqrt{r^{2}-x^{2}}}
$$


where $r^{2}=x^{2}+z^{2}, R$ - the radius of the cross section of the considered zone, $n(r, y)$ - refractive index at a distance of $r$ from the axis of the torch. Thus, the value of the phase function $\Delta \psi$ can be restored by formula (8), knowing $n_{0}$.

The composition of dry air (relative molar concentrations of dry air components), as well as the dependence of the saturated vapor pressure on temperature, must be known to calculate the refractive index of the medium (air) $n_{0}$ unperturbed by the flame.

Atmospheric pressure is equal to the sum of the partial pressures of the dry air components and the partial pressure of water vapor. The partial pressure of water vapor in the mixture (air) is determined by the formula for the relative humidity of air:

$$
P_{W}=\frac{\varphi_{W} \cdot P_{0 W}}{100},
$$

where $P_{W}$ is the partial pressure of water vapor, $P_{0 W}$ is the saturated vapor pressure at a given temperature, $\varphi_{W}$ is the relative air humidity in the room.

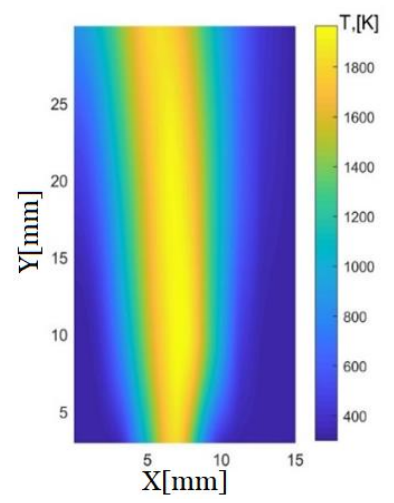

Fig. 4. The recovered radial temperature field of hydrogen-air flame.

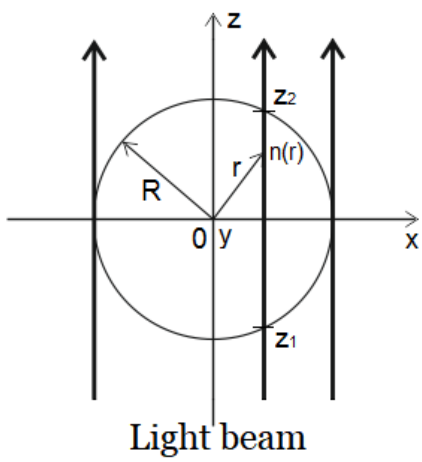

Fig. 5. The section of the investigated axisymmetric object in the plane $y=$ const. 
The molar concentration of water vapor in the mixture (air) can be determined by the equation of state of an ideal gas, knowing the value of $P_{W}$ :

$$
v_{W}=\frac{P_{W}}{R_{f} \cdot T_{0}}
$$

where $R_{f}$ is the universal gas constant, $T_{0}$ is the room temperature. Similarly, we calculate the value of the molar concentration of the entire mixture (air):

$$
v=\frac{p_{0}}{R_{f} \cdot T_{0}}
$$

where $p_{0}$ is atmospheric pressure (in the room).

Then the relative molar concentration of water vapor in the air:

$$
C_{W}=\frac{v_{W}}{v}
$$

The refractive index $n_{0}$ of the medium unperturbing by the flame, taking into account (6) and (9) - (12), will be determined as

$$
n_{0}=1+\frac{p_{0}}{p_{n . c .}} \frac{T_{n . c .}}{T_{0}}\left[\left(\sum_{k} A_{k}\left(1+\frac{B_{k}}{\lambda^{2}}\right)\right)\left(1-C_{W}\right)+\left(A_{W}\left(1+\frac{B_{W}}{\lambda^{2}}\right)\right) C_{W}\right],
$$

where $p_{\text {n.c. }}$ - atmospheric pressure under normal conditions $\left(101.325 \cdot 10^{3} \mathrm{~Pa}\right) ; T_{\text {n.c. }}$ temperature under normal conditions $\left(0^{\circ} \mathrm{C}\right) ; A_{k}$ and $B_{k}$ - dispersion coefficients for the $k$ th component of dry air; $A_{W}$ and $B_{W}$ - dispersion coefficients for water; $\lambda$ is the wavelength of the radiation source.

The radial field of the refractive index calculated by formula (6) is shown in Fig. 6.a. The conditions were accepted in the calculation: $T_{0}=27^{\circ} \mathrm{C}, \lambda=0.630 \mu \mathrm{m}$, $p=p_{0}=101.325 \cdot 10^{3} \mathrm{~Pa}$ (normal conditions), $A_{k}$ and $B_{k}$ are the values taken from the reference data.

The phase function must be reconstructed from the hilbertogram according to the algorithm proposed in [2], and the refractive index of the medium must be determined by determing the Abel equation to solve the inverse problem (reconstruction of the flame temperature from the hilbertograms). The calculated inverse field of the phase function (for the wavelength $\lambda=0.630 \mu \mathrm{m}$ ), obtained by calculating the Abel integral (8), is shown in Fig. 6.b.

The refractive index depends on temperature and 4-x values of mole fraction of combustion products, which follows from formula (6). Measurements at three different wavelengths of the probing field are sufficient to determine all unknown parameters, since the combustion area is divided into zones consisting of 3 components of combustion products.

The radial profiles of temperature, molar concentrations of combustion products, refractive index and the corresponding values of the phase function, hilbertogram and interferogram are shown in Fig. 7 for the section $y=10 \mathrm{~mm}$ and $\lambda=0.630 \mu \mathrm{m}$.

The numerically simulated hilbertograms obtained from the calculated phase function $\Delta \psi$ for $\lambda=0.630,0.520,0.405 \mu \mathrm{m}$, respectively, are shown in Fig. 8 . The dispersion coefficients were taken from the reference data, and the relative air humidity in the room was taken $\varphi_{W}=60 \%$ in the numerical simulation of the hilbertograms. 

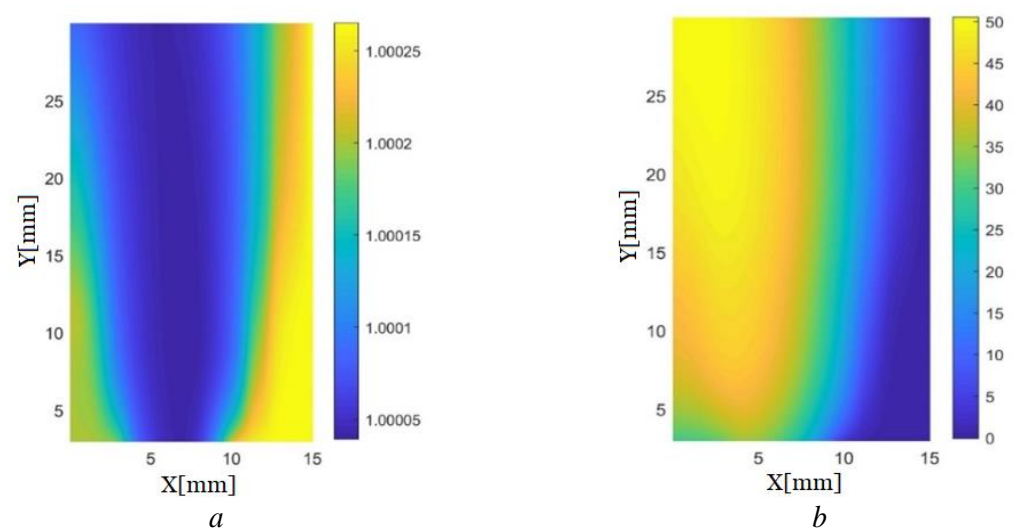

Fig. 6. $(a)$ - radial field of the refractive index of a hydrogen-air flame $(\lambda=0.630 \mu \mathrm{m}) ;(b)$ - field of the phase function for a radius $r$ from 0 to $15 \mathrm{~mm} \lambda=0.630 \mu \mathrm{m}$.
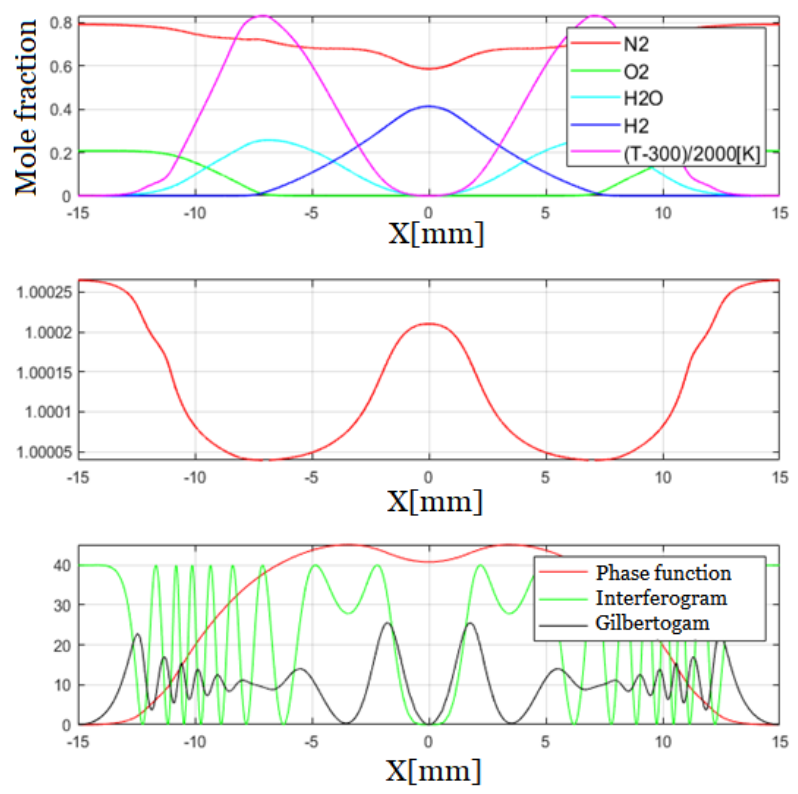

$a$

$b$

Fig. 7. (a) - radial profiles of temperature $T$ and of molar concentrations $C_{k} ;(b)$ - radial profile of the refractive index $n(r, y) ;(c)$ - phase function, interferogram and hilbertogram $(y=10 \mathrm{~mm}$, $\lambda=0.630 \mu \mathrm{m})$.

The results obtained confirm the feasibility of multi-wave optical diagnostics in direct and inverse problems of studying phase structure and temperature fields in an axisymmetric flame. 
Optical Diagnostics of Temperature and Structural Parameters of an Axisymmetric Flame 9
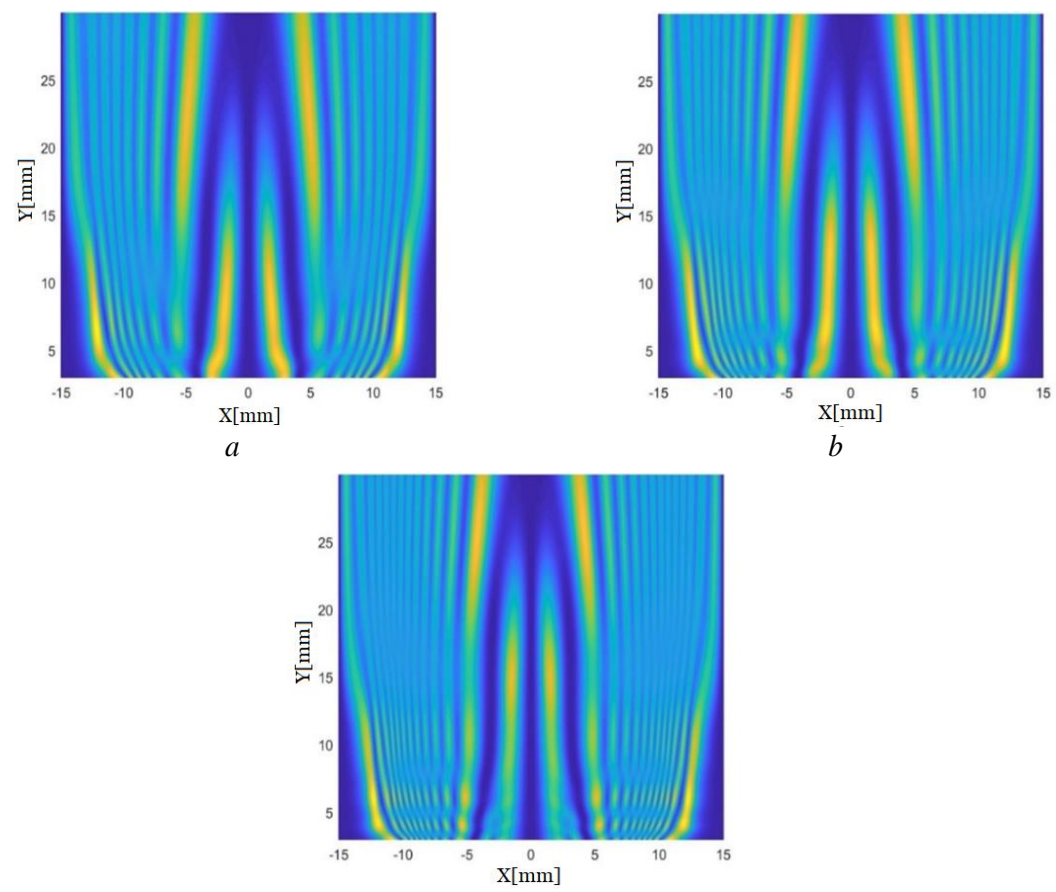

$c$

Fig. 8. Hilbert images numerically modeled by the field of the phase function $\Delta \psi$ for $r$ from -15 to $15 \mathrm{~mm}:(a)-y=0.630 \mu \mathrm{m} ;(b)-y=0.520 \mu \mathrm{m} ;(c)-y=0.405 \mu \mathrm{m}$.

\section{Conclusion}

The study of a hydrogen-air flame was done in the presented work using the methods of Hilbert optics and numerical modeling in the approximation of axial symmetry of the flame using the Abel integral. The reliability of the results is confirmed by comparing the hilbertograms obtained in the experiment (Fig. 1.a) and reconstructed from the phase structure according to the Abel equation (Fig. 8). The comparison results are used as a quality criterion of the modeling the phase structure and temperature field using the Abel transform in the study of the combustion process. The application of the developed method for solving the inverse problem - restoration of the temperature field from the Hilbert image of the phase structure of a hydrogen-air flame will become the next stage of work.

The work was done within the framework of the state assignment of IT SB RAS project III.18.2.5 (state registration No. AAAA-A17-117030310010-9, No. AAAAA17-117022850021-3), partial financial support from RFBR (project No. 20-3890195). 
10 V. Arbuzov, E. Arbuzov, Y. Dubnishchev, O. Zolotukhina, V. Lukashov

\section{References}

1. Qunxing Huang, Fei Wang, Jianhua Yan, and Yong Chi.: Simultaneous estimation of the 3-D soot temperature and volume faction distributions in asymmetric flames using highspeed stereoscopics images. Applied Optics 51(15), 2968-2978 (2012).

2. Dreyer, J.A. H., Slavchov, R.I., Rees, E.J. et al.: Improved methodology for performing the inverse Abel transform of flame images for cilir ratio pyrometry. Applied Optics 58(10), 2662-2670 (2019).

3. Dubnishchev, Yu.N., Arbuzov, V.A., Lukashov, V.V., Sharov, K.A., Lemanov, V.V.: Optical Hilbert Diagnostics of Hydrogen Jet Burning. Optoelectron., Instrum. Data Process 55(1), 16-19 (2019).

4. Litvinenko, Yu.A.: Ustoychivost' dozvukovykh makro- i mikrostruynykh techeniy i mikrostruynoye goreniye (obzor) [Stability of subsonic macro- and microstructural flows and microjet combustion (review)]. Sibirskiy fizicheskiy zhurnal [Siberian Physical Journal] 12(3), 83-89 (2017).

5. Belozerov, A.F.: Opticheskie metody vizualizacii potokov [Optical Methods of Gas Flow Visualization]. Izd. Kazan. Gos. Tekh. Univ., Kazan (2007).

6. Arbuzov, V.A., Arbuzov, E.V., Dubnishchev, Yu.N., Zolotukhina, O.S., Lukashov, V.V.: Recovery of the Temperature Field in the Case of Combustion of Premixed Propane-Air Mixtures by Methods of the Hilbert Optics. Optoelectron., Instrum. Data Process 56(1), 74-82 (2020).

7. Ioffe, B.V.: Refraktometricheskiye metody khimii [Refractometric methods of chemistry]. 3rd ed. Rev. L.: Chemistry, Leningrad department (1983).

8. Hauf, V., Grigul, U.: Opticheskiye metody v teploperedache [Optical methods in thermal transport]. Mir, Moscow (1973).

9. Toro, V.V., Mokhov, A.V., Levinsky, H.B., Smooke, M.D.: Combined experimental and computational study of laminar, axisymmetric hydrogen-air diffusion flames. Proceedings of the Combustion Institute 30, 485-492 (2005). 